\title{
Biomedical optics centers: forty years of multidisciplinary clinical translation for improving human health
}

Bruce J. Tromberg

R. Rox Anderson

Reginald Birngruber

Ralf Brinkmann

Michael W. Berns

John A. Parrish

Gabriela Apiou-Sbirlea 


\title{
Biomedical optics centers: forty years of multidisciplinary clinical translation for improving human health
}

\author{
Bruce J. Tromberg, ${ }^{\mathrm{a}, *}$ R. Rox Anderson, ${ }^{\mathrm{b}, \dagger}$ Reginald Birngruber, ${ }^{\mathrm{b}, \mathrm{c}, \dagger}$ Ralf Brinkmann, ${ }^{\mathrm{c}}$ Michael W. Berns, ${ }^{\mathrm{a}}$ \\ John A. Parrish, ${ }^{d}$ and Gabriela Apiou-Sbirleab,* \\ aUniversity of California, Irvine, Beckman Laser Institute, 1002 Health Sciences Road East, Irvine, California 92612, United States \\ ${ }^{b}$ Wellman Center for Photomedicine and Harvard Medical School, Massachusetts General Hospital, Research Institute, Department of \\ Dermatology, 40 Blossom Street, Boston, Massachusetts 02114, United States \\ 'Medical Laser Center Lübeck, Peter Monnik Weg 4, Lübeck 23562, Germany \\ ${ }^{\mathrm{d} C e n t e r}$ for Integration of Medicine and Innovative Technology, 125 Nashua Street, Boston, Massachusetts 02114, United States
}

\begin{abstract}
Despite widespread government and public interest, there are significant barriers to translating basic science discoveries into clinical practice. Biophotonics and biomedical optics technologies can be used to overcome many of these hurdles, due, in part, to offering new portable, bedside, and accessible devices. The current JBO special issue highlights promising activities and examples of translational biophotonics from leading laboratories around the world. We identify common essential features of successful clinical translation by examining the origins and activities of three major international academic affiliated centers with beginnings traceable to the mid-late 1970s: The Wellman Center for Photomedicine (Mass General Hospital, USA), the Beckman Laser Institute and Medical Clinic (University of California, Irvine, USA), and the Medical Laser Center Lübeck at the University of Lübeck, Germany. Major factors driving the success of these programs include visionary founders and leadership, multidisciplinary research and training activities in light-based therapies and diagnostics, diverse funding portfolios, and a thriving entrepreneurial culture that tolerates risk. We provide a brief review of how these three programs emerged and highlight critical phases and lessons learned. Based on these observations, we identify pathways for encouraging the growth and formation of similar programs in order to more rapidly and effectively expand the impact of biophotonics and biomedical optics on human health. ๑ 2016 Society of Photo-Optical Instrumentation Engineers (SPIE) [DOI: 10.1117/1.JBO.21.12.124001]
\end{abstract}

Keywords: translational research origins; biophotonics; biomedical optics; clinical problem driven innovation; multidisciplinary research.

Paper 2016-1108-TransBio received . NaN, ; accepted for publication . NaN, ; published online Dec. 20, 2016.

\section{Introduction}

The annual impact of photonics on the U.S. economy is estimated at $\$ 3$ trillion, with biophotonics a fast-growing $\sim 90$ billion/year (worldwide) component. ${ }^{1,2}$ However, the present robust commercial activity in biophotonics was, by comparison, practically nonexistent in the late 1970s and early 1980s when the Wellman Center for Photomedicine (WCP, Mass General Hospital, USA), the Beckman Laser Institute and Medical Clinic (BLI, University of California Irvine, USA), and the Medical Laser Center Lübeck (MLL at the University of Lübeck, Germany) were in formative states. The emergence of these and other multidisciplinary centers for lasers and optics in biology and medicine over the past 30 to 40 years is a remarkable success story that parallels the growth of a variety of medical photonics technologies spanning from medical lasers to endoscopes, and optical coherence tomography (OCT) to nearinfrared oximetry. This article is not intended to be a comprehensive review of all centers that have played a key role in this worldwide expansion. Rather we highlight three complementary programs with roots going back to the mid-late 1970s that emerged and grew under similar circumstances with colocated clinical and technology development activities.

*Address all correspondence to: Bruce J. Tromberg, E-mail: bjtrombe @uci.edu; Gabriela Apiou-Sbirlea, E-mail: gapiou@mgh.harvard.edu

†These authors have equally contributed.
Biophotonics and biomedical optics naturally brings together collaborative multidisciplinary teams who regularly synthesize concepts from science, engineering, and medicine to develop new diagnostic and therapeutic methods and devices. Centers like WCP, BLI, and MLL have played a critical role in providing space, facilities, and opportunities that have led to remarkably productive "bench-to-bedside" activity and impact, much of which began as innovations motivated by important, unsolved clinical problems. This "translational research" concept has received significant attention recently, as evidenced in the USA by the emergence (in 2012) of the National Center for Advancing Translational Sciences (NCATS) at the National Institutes of Health (NIH). The goal of this 685 million/ year Institute is to "transform the translational science process so that new treatments and cures for disease can be delivered to patients faster." ${ }^{3}$ While interest in translation is clearly gaining worldwide attention, it is instructive to note that MLL, WCP, BLI, and similar centers have pioneered these activities for more than 35 years. Biophotonics and biomedical optics naturally span across domains of basic research, technology development, commercialization, and clinical translation. The emergence of commercially successful medical photonics technologies is an achievement that parallels the growth of these major biophotonics research centers. Understanding aspects of their formation is essential not only for ensuring the continued clinical translation of enabling technologies, but for maintaining a robust pipeline 
for generating new ideas, technologies, and workforce that will advance these activities for the benefit of human health.

WCP, MLL, and BLI are well known for major research and commercial activities in the development of new optics and photonics technologies that advance both medical therapeutics and diagnostics. Examples of therapeutic methods include selective cutaneous, ophthalmic, and gastrointestinal laser treatments based on fundamental light-tissue interaction mechanisms that span photothermal, photochemical, and photomechanical regimes. Light-based methods are widely used in medicine with annual commercial sales of phototherapeutic lasers and similar light sources estimated to be approximately $\sim 1 \mathrm{~B} /$ year, creating a much larger overall economic stimulus from medical services. An important common characteristic of these three centers is their continued commitment to photomedicine for improving patient outcomes. This has helped to create a patient oriented culture in programs that include faculty, staff, and students with diverse training in multiple subspecialties of medicine, science, and engineering. Each year, millions of patients are treated worldwide using biophotonics technologies. In addition, thousands of patients are treated and enrolled in Institutional Review Board (IRB)-approved protocols by WCP, BLI, and MLL associated physicians. Optical technologies born in these centers are now the standard of care for dozens of medical problems and diseases.

Each center has also pioneered a diverse collection of diagnostic technologies based on the fundamental principles of tissue spectroscopy and imaging. Optical diagnostics are well suited for noninvasive and minimally invasive, point-of-care medicine. The current medical photonics diagnostic market is primarily based on optical endoscopy, microscopy, near-infrared tissue/ pulse oximetry, perfusion/flow imaging, and OCT. The field of biophotonics and biomedical optics has been dominated by remarkable advances in these and related devices over the past $\sim 25$ years. Factors, such as miniaturization, reductions in barriers-to-access, and the changing healthcare landscape, will further accelerate the growth and commercialization of diagnostic medical photonics. In addition, multimodality and hybrid approaches, spanning from "optics + ultrasound" to "optics + MRI" are rapidly expanding and expected to achieve clinical and commercial success in the coming years. Many of these methods are described in this special issue. BLI, MLL, and WCP have each played a unique role in fostering translation of these methods into medical practice, because of their longstanding commitment to patient outcome and special expertise in identifying new and clinically impactful applications of emerging technologies. Examples include advancing OCT in vascular, pulmonary, GI, and head and neck disease, and using diffuse optics to guide chemotherapy in breast cancer. This is possible, in part, because of each center's rich portfolio and well-established infrastructure that supports time and effort-intensive translational biophotonics studies. Overall, WCP, BLI, and MLL have a portfolio of more than 50 Institutional Review Board (IRB) protocols in optical diagnostics, and enroll hundreds of patients annually on multiple investigator-initiated hypothesis-based studies.

\section{Centers}

\subsection{Wellman Center for Photomedicine}

The Wellman Center for Photomedicine (WCP) is one of the five thematic research centers located in Mass General Hospital, the academic hospital of Harvard Medical School. Founded in 1976 by Harvard Dermatology Professor John A. Parrish MD, WCP occupies today 38,000 sq. ft, and has 14 core faculty, 12 affiliated faculty, and a total of more than 250 professors, staff, and students working in a multidisciplinary clinical translational environment. Its name honors Arthur Wellman, a philanthropist whose wife was successfully treated for disabling psoriasis with oral psoralen activated by UV-A radiation (PUVA), a new discovery at the time by Dr. Parrish whose early work focused on human skin photobiology and novel treatments for skin disease (Fig. 1). Dr. Parrish then recruited physicists, engineers, pathologists, immunologists and cell biologists to work together investigating the fundamentals of light-tissue interactions. The original motivation was to explain and improve treatments that clinical research had already shown to be effective.

While this ambitious goal was partially achieved, the effort led to a much wider impact. WCP multidisciplinary research teams developed a passion for understanding and manipulating the rich portfolio of molecular changes caused by optical irradiation of living tissues. Fascinating photochemical, thermal, and physical reactions were explored and documented in detail. Simultaneously, laser, fiber optic, computational, imaging, and molecular biological technologies were rapidly advancing. In 2004, WCP formally embraced the power of multidisciplinary research. In contrast to traditional medical departments based mainly on organ systems, WCP became a nondepartmental MGH Thematic Center, with Dr. Rox Anderson MD as its new director.

What started as a skin laboratory evolved to be a center contributing high-impact solutions for "the skin and everything in it." Laser lithotripsy was developed and launched commercially for removing urinary stones. ${ }^{4}$ Other projects such as laser angioplasty met with scientific success but practical failure. The concept of selective photothermolysis was introduced, ${ }^{5}$ leading to many novel laser treatments for skin, eye, and laryngeal disorders. For the first time, biomedical needs spearheaded the invention of new laser technologies. Fractional laser treatment was invented to stimulate skin remodeling, and is fast becoming a major treatment to normalize scars. The early interest in lightactivated drugs evolved into creative, versatile examples of

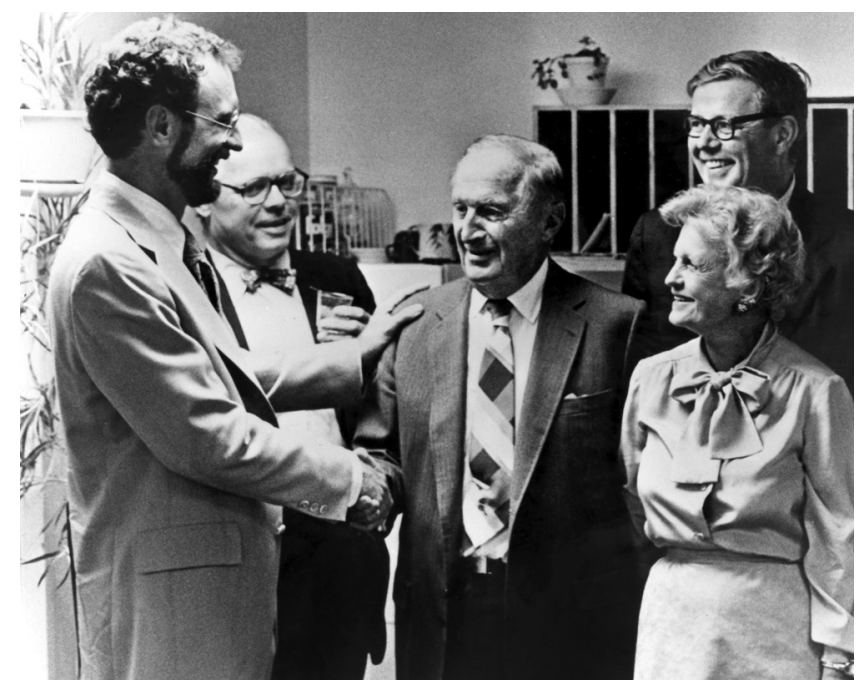

Fig. 1 Founding WCP director Dr. John Parrish (left), meets with philanthropists Arthur (center) and Gullan Wellman (front right) in 1982. Also present are Thomas B. Fitzpatrick, Chair of the Department of Dermatology, Harvard Medical School and MGH (second from left) and Nicolas W. Thorndike, Chairman of MGH Board (second row, right). 
photodynamic therapy (PDT). Benzoporphyrin derivative, a drug initially intended for cancer treatment, was repurposed to become the first approved treatment for retinal macular degeneration. ${ }^{6}$ Studies of tissue optics including early mathematical models of diffuse radiation transfer were produced, which set the stage for optical diagnostics. The first laser confocal microscope for human use was invented and commercialized, ${ }^{7}$ now in clinical use. Optical diagnostics became a sophisticated and diverse field unto itself. The speed, resolution, and capabilities of OCT, which was invented at MIT almost 30 years ago for retinal imaging, were greatly advanced by WCP innovations. ${ }^{8}$ Unprecedented 3-D tissue imaging became possible through needles, cardiac catheters, swallowed microscopes, and other devices. In addition to the stand-alone optical diagnostic devices in many medical specialties, we are witnessing now their integration with conventional medical imaging, their use for real-time guidance of surgical and other procedures, and low-cost simplified versions applicable to solving global health problems.

The fundamental strength of WCP has always been highquality scientific inquiry. For example, a basic understanding of photothermal processes is what enabled a host of new laser therapies. Tissue optics and in vivo spectroscopy enabled optical diagnostics. Photochemistry enabled photodynamic therapies, including some surprising capabilities now in development. New themes are emerging at WCP, sometimes beyond the general theme of photomedicine. For example, the popular use of controlled cryotherapy for selective removal of unwanted body fat is a WCP innovation.

\subsection{Beckman Laser Institute and Medical Clinic}

Around the same time that Dr. Parrish was working on human skin photobiology, Dr. Michael W. Berns, $\mathrm{PhD}$, was a professor of cell biology at the University of California, Irvine (UCI). In 1979, Dr. Berns invited Dr. Arnold O. Beckman, PhD, an inventor-businessman-philanthropist and founder of Beckman Instruments, Inc. to an "open house" in the Berns lab. At that time, Dr. Berns had established the first NIH P41 National Biotechnology Research Resource Center for laser microbeams [the Laser Microbeam Program (LAMP)], a new technology for cellular microsurgery he had pioneered as a Cornell University graduate student. ${ }^{9}$ Lasers and their potential applications in biology and medicine captured the imagination of Dr. Beckman and he worked with Dr. Berns to form the Beckman Laser Institute and Medical Clinic (BLI) in 1982 (Fig. 2). Together they created a BLI nonprofit foundation to raise money and build a free-standing building on the UCI campus. This early successful example of a public-private partnership led to the original BLI structure that opened in 1986. In 2003, Dr. Bruce Tromberg, PhD, was appointed BLI director and Dr. J. Stuart Nelson, MD, medical director. BLI was designated as a campuswide "special research program" in 2006 but maintains its original Medical School affiliation as a division in the Department of Surgery.

Although the terminology did not exist at the time, this was the first dedicated translational facility constructed specifically for advancing basic science, technology development, and clinical translation of lasers and optics, all under one roof. Several unique design features that were decades ahead of their time characterized the building, including open shared spaces; shared resources for cell biology, biochemistry, histopathology, animal models, and image processing; and a dedicated laser clinic and

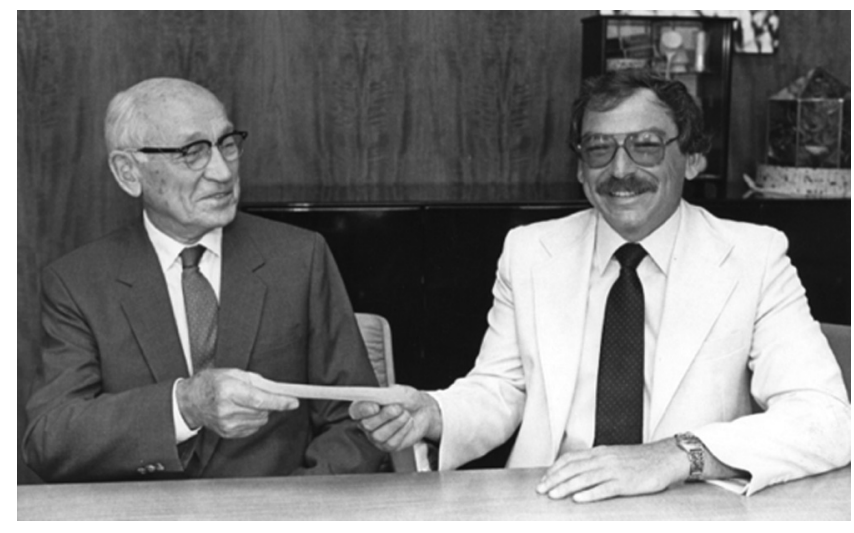

Fig. 2 In 1982 Dr. Arnold O. Beckman (left) presented Dr. Michael W. Berns, founding BLI director, with the first check to establish the Beckman Laser Institute at UC Irvine.

operating room. In addition, a fiber optic network was built throughout the BLI clinic for delivering light from massive, water- and power-hungry lasers, the standard of the day, to any treatment room in the clinic. Although UCI and Lasers were only 17 and 22 years old, respectively, when BLI was established, Dr. Beckman had the foresight to invest significant resources in a young professor, an unproven university, and an emerging technology.

The goal of the 1979 NIH LAMP facility was to develop tools for selectively altering small regions of living cells in order to study cell and organelle function. As core technologies continued to develop, new applications of the laser microbeam evolved, particularly in the area of gradient force optical trapping, chromosome microdissection, laser-induced gene transfection, and nonlinear laser microbeams. ${ }^{10}$ Although rooted in basic science, the perceived clinical and commercial potential of LAMP technologies inspired Dr. Beckman and Dr. Berns to establish the BLI, a crucial step that accelerated the clinical translation of lasers and optics.

Today the BLI is a 37,000 sq.- $\mathrm{ft}$ free-standing facility with 17 faculty and more than 200 professors, staff, and students from more than 10 science, engineering, and clinical departments on the UC Irvine campus. Clinical applications emerged within BLI in the late 1980s, particularly in the areas of PDT for cancer and dynamic cooling technology for cutaneous laser surgery. ${ }^{11}$ Several important new technologies, biomedical applications, and clinical studies appeared during subsequent years, including laser microbeam assisted in vitro fertilization technologies, ${ }^{10}$ Doppler OCT for imaging blood flow, ${ }^{12}$ diffuse optical methods for imaging perfusion and metabolism in thick tissues, ${ }^{13}$ and multimodality endoscopic tomographies. ${ }^{14}$ Virtual photonics technologies also emerged, an effort entirely dedicated to biophotonics modeling and computation for simulating and visualizing light propagation in biological tissues.

\subsection{Medical Laser Center Lübeck}

In Europe in 1975 Dr. Franz Hillenkamp PhD, a young biomedical engineer who led the Laboratory for Coherent Optics at the "Gesellschaft für Strahlen- und Umweltforschung," a National Laboratory for Radiation and Environmental Research in Munich, Germany, introduced a Medical Laser Applications Laboratory. This center provided standardized laser- and optical delivery-technology together with extended animal facilities for 
application oriented in vitro and in vivo studies in laser medicine. Medical scientists from ophthalmology, gastroenterology, urology, neurosurgery, and oral surgery performed studies in the areas of photocoagulation and photodisruption in ophthalmology, laser-induced hemostasis in gastric ulcers, laser therapy of bladder tumors, and laser bone surgery. Dr. Alfons Hofstetter MD, the urologist in this research group, left Munich and became Chairman of Urology at the University of Lübeck in 1984. Much like Dr. Parrish and Dr. Berns whose early research seeded the growth of larger centers, Dr. Hofstetter's work initiated the foundation of the Medical Laser Center Lübeck (MLL-formal "Medizinisches Laserzentrum Lübeck GmbH"). The MLL, funded by the German state of Schleswig-Holstein as a separate, but university-associated R\&D Institution at the University of Lübeck, opened in 1986.

The intention of MLL from the very beginning was to introduce, launch, and establish laser technologies in medicine. Core expertise in high-power IR-lasers, short- and ultra-short pulselasers, miniaturized solid-state lasers, etc. was leveraged by interdisciplinary and transinstitutional collaborations between research institutions, industry, and hospitals. In order to facilitate translation of research to clinical practice, the MLL was founded as a nonprofit limited liability company $(\mathrm{GmbH})$ with seven associates, two universities including the leading state hospital, four biomedical companies, and the Hanseatic city of Lübeck. Physicists, a biologist, engineers, and physicians were hired and a research building was constructed together with a guesthouse for collaborators and participants of conferences and training courses. Application-oriented R\&D-projects in urology (lithotripsy), tumor therapy (PDT) quickly emerged.

In 1991, Dr. Reginald Birngruber PhD, MD became the Chief Executive Officer (CEO) and Chief Research Officer (CRO) of the MLL and research initiatives in ophthalmology (picosecond photodisruption, PDT, microphotocoagulation) and cardiovascular laser therapy (laser angioplasty) were added, always in close collaboration with clinicians and various laser companies. Understanding mechanisms of light-tissue interaction and photothermal tissue effects became crucial research activities that helped drive the development of novel and economically successful technologies. Examples include, mathematical modeling of temperature profiles, thermal denaturation kinetics,${ }^{15}$ and nonlinear processes in pulsed laser tissue ablation. ${ }^{16}$ New laser systems like the Alexandrite solid-state laser and high-power picosecond lasers were modified and adapted to solve specific clinical problems. A mutual collaboration with the Wellman Center for Photomedicine in Boston resulted in the invention and development of new medical laser applications, including carrier-mediated PDT to make age-related macula degeneration (AMD) a treatable disease for the first time ${ }^{17}$ and selective retina therapy for precise treatments of various early stage retinal diseases. ${ }^{18}$ Several medical laser devices were commercialized in collaboration with MLL's partners, for example, the first feedback controlled laser-lithotriptor Lithognost, ${ }^{19}$ several PDT laser devices for ophthalmology, and a miniaturized handheld IR-laser scalpel for trabeculectomy.

In 2005, the Institute of Biomedical Optics (BMO) was formed out of the MLL-research teams and became part of the University of Lübeck. BMO was created to offer new courses in biomedical optics, photonics, optical imaging and laser medicine to educate and prepare the next generation for emerging technologies in optical life sciences. BMO, now an academic institution, hosts undergraduate and graduate students and can

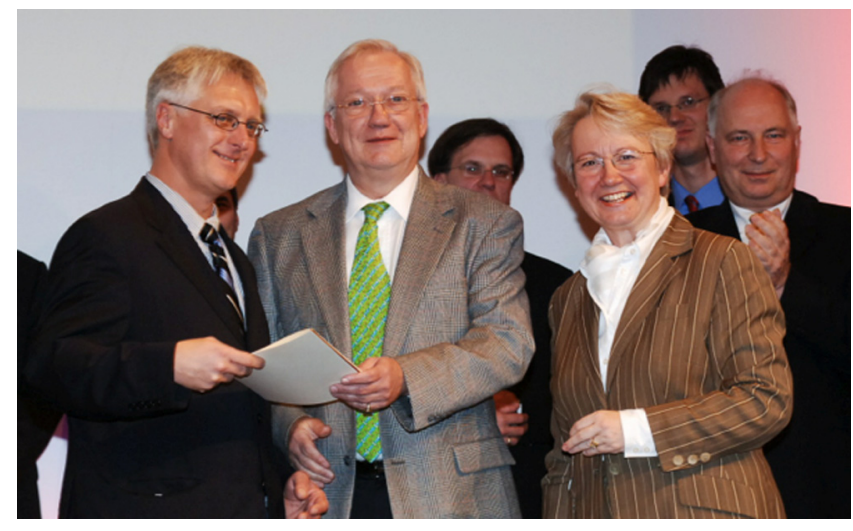

Fig. 3 Dr. Reginald Birngruber (center), MLL founding CEO, meets with current CEO Ralf Brinkmann (left) and Annette Schavan (right front), German Minister of Research and Education, in 2006.

conduct research in all aspects of optics in medicine and biology, supported by more traditional state and federal funding. MLL kept its status as a nonprofit R\&D company financially independent and supported solely by project funds derived from clinical, translational and applied research in collaboration with clinical and industrial partners (Fig. 3). After Dr. Birngruber retired in 2010, Dr. Alfred Vogel PhD became the Director of the BMO, and Dr. Ralf Brinkmann PhD took over MLL as its CEO in 2011.

Today, MLL and BMO have 10 faculty and more than 80 professors, staff, and students working in the interdisciplinary field of biophotonics. Therapeutic laser applications are still the strongest MLL focus area. Activities in imaging and in vivo diagnostics, metrology, laser material processing and prototype development, and management of clinical studies continue to expand. A unique feature of MLL as a limited liability company is that it can legally register prototypes according to the European medical product law and perform proof-of-concept clinical trials. This strong partnership between a university institute, a university hospital, and a highly flexible nonprofit R\&D company, sharing infrastructure and personnel, but being formally independent, has been proven highly successful. Importantly, the MLL with its own lean administrative structure and low overhead can act quickly and adapt to short-term industrial tasks, but also serve as a reliable partner in long-term public funded programs.

\section{Common Features for all Centers}

What common strategies foster "translational" research in these centers, all the way from early discovery, through development, commercialization, and adoption in medical practice? First, all are either located in or closely affiliated with academic medical centers and medical schools. In contrast to traditional medical, engineering, and basic science departments based mainly on organ systems or specific disciplines, all are transdepartmental centers with faculty, staff, and students who have different backgrounds and perspectives. Intellectual diversity, in turn, plays a key role in the second major common feature: each center is dedicated to the idea that optics and photonics technologies can be used to advance science for solving practical problems that impact human health. With multidisciplinary clinics and physicians embedded in each center, collaborating scientists and engineers are able to develop a "translational vision" focused on identifying and solving important clinical problems. In addition, 
there are unique resources available to help investigators develop IRB-approved clinical protocols and enroll patients on investigational studies that use biophotonics technologies. This culture is strongly reinforced by the emphasis in each center on developing new medical laser therapies. Millions of people have benefited from treatments launched directly from WCP, BLI, and MLL. Opportunities to continue this activity and develop new approaches including image-guided therapies are considered to be highly motivating challenges.

Although located in different regions spanning private (WCP), public (BLI), and mixed (MLL) operational models, each has embraced supporting high-risk, high-reward research. This fourth common element is made possible, in part, by having a diverse funding portfolio that includes a balance of extramural peer-reviewed grants that are restricted to specific projects, and more flexible sources of exploratory funding derived from royalty revenues, philanthropy, and other commercialization activities. These funds can be used to seed emerging ideas, initiate new lines of study, and develop proof-of-concept technologies and prototypes. Leveraging with collaborating departments and schools has also proven successful. In the case of WCP and BLI, US Department of Defense (DOD) support is an important mechanism that is synergistic with NIH since it is aimed to solve military medical problems using photomedicine strategies. The problem-motivated, get-the-jobdone culture of DOD is a powerful stimulus for hatching and developing new solutions for combat medical problems. Many of these can be "dual use," helping both civilian and military patients. Importantly, the military Medical Photonics Program administered by the Air Force Office of Scientific Research (AFOSR) has had enormous impact on the growth of biophotonics and biomedical optics worldwide, both through ongoing programs at these centers, and via direct support of research at other institutions. Despite the diversity and growth of research support at these centers, funding still limits the volume and rate of research.

In summary, MLL, WCP, and BLI are privileged to be in problem-rich medical environments with academically and intellectually diverse faculty, students, and staff. Each has a strong tradition of problem solving, innovation, and technology development that has contributed to the worldwide growth of medical photonics technologies and translational research. With continued global expansion of photonics technologies, scientists and engineers will have even more opportunities to partner with physicians and patients to ensure that new discoveries are rapidly translated into cost-effective strategies for curing and preventing disease. Biophotonics centers have a well-established history for accelerating this process, making their unique interdisciplinary problem-solving mission even more compelling and essential than ever.

\section{Disclosures}

No conflicts of interest, financial or otherwise, are declared by the authors.

\section{References}

1. National Research Council, Optics and Photonics: Essential Technologies for Our Nation, The National Academies Press, Washington, DC (2013).

2. G. Lee et al., "A study of biophotonics: market segments, size and growth," Opt. Photonik 2, 30-35 (2007).
3. F. Collins, "Reengineering translational science: the time is right," Sci. Trans. Med. 3, 90cm17 (2011).

4. G. Watson et al., "The pulsed dye laser for fragmenting urinary calculi," J. Urol. 138, 195-198 (1987).

5. R. R. Anderson and J. A. Parrish, "Selective photothermolysis: precise microsurgery by selective absorption of pulsed radiation," Science $\mathbf{2 2 0}$, 524-527 (1983).

6. U. Schmidt-Erfurth et al., "Vascular targeting in photodynamic occlusion of subretinal vessels," Ophthalmology 101(12), 1953-1961 (1994).

7. R. H. Webb and G. W. Hughes, "Detectors for video rating scanning images," Appl. Opt. 32, 6227-6235 (1993).

8. J. F. de Boer et al., "Improved signal-to-noise ratio in spectral-domain compared with time-domain optical coherence tomography," Opt. Lett. 28(21), 2067-2069 (2003).

9. M. W. Berns et al., "Laser microsurgery in cell and developmental biology," Science 213, 505-513 (1981).

10. M. W. Berns et al., "Laser scissors and tweezers," in Methods in Cell Biology, M. P. Sheetz Ed., Vol. 55, pp. 71-98, Academic Press, Cambridge, Massachusetts (1997).

11. J. S. Nelson et al., "Dynamic epidermal cooling during pulsed laser treatment of port-wine stain: a new methodology with preliminary clinical evaluation," Arch. Dermatol. 131(6), 695-700 (1995).

12. Z. Chen et al., "Optical Doppler tomographic imaging of fluid flow velocity in highly scattering media," Opt. Lett. 22, 64-66 (1997).

13. R. C. Haskell et al., "Boundary conditions for the diffusion equation in radiative transfer," J. Opt. Soc. Am. A 11, 2727-2741 (1994).

14. J. Yin et al., "Integrated intravascular optical coherence tomography ultrasound imaging system," J. Biomed. Opt. 15(1), 010512 (2010).

15. R. Birngruber, F. Hillenkamp, and V. P. Gabel, "Theoretical investigations of laser thermal retinal injury," Health Phys. 48(6), 781-796 (1985).

16. A. Vogel and V. Venugopalan, "Mechanisms of pulsed laser ablation of biological tissues," Chem. Rev. 103, 577-644 (2003).

17. TAP Study Group, "Photodynamic therapy of subfoveal choroidal neovascularization in age-related macular degeneration with verterporfin," Arch. Ophthalmol. 117, 1329-1345 (1999).

18. J. Roider et al., "Microphotocoagulation: selective effects of repetitive short laser pulses," Proc. Natl. Acad. Sci. U. S. A. 90, 8643-8647 (1993).

19. H. Neuhaus et al., "Endoscopic lithotripsy PF bile duct stones using a new laser with automatic stone recognition,' Gastrointest. Endosc. 40(6), 708-715 (1994).

Bruce J. Tromberg is a professor of biomedical engineering and surgery at the University of California, Irvine (UCI) and Director of UCl's Beckman Laser Institute and Medical Clinic. He has more than 25 years of experience in the development of optics and photonics technologies and their application to biology and medicine. His research interests are in the development of quantitative, broadband biophotonics technologies for characterizing and imaging tissue structure, function and composition across spatial scales.

R. Rox Anderson is a professor in dermatology at Harvard Medical School, and director of the Wellman Center for Photomedicine at Massachusetts General Hospital. He conceived and codeveloped the concept of microscopic target-selective laser therapy. Lasers now in widespread use for pediatric portwine stains, pigmented lesions, tattoos, and hair removal came from this work. He coinvented the confocal laser scanning microscope, fractional laser treatment, and selective cryolipolysis, while also contributing to the development of lasers for lithotripsy, cardiovascular, and eye diseases.

Reginald Birngruber studied electrical engineering and physics, and graduated in physics (PhD) and medical biophysics (MD habilis) in Germany. After his retirement from CEO and CRO of the Medical Laser Center Luebeck and the chair of the Institute of Biomedical Optics at the University Luebeck, Germany, he now makes his expertise, and contacts available to projects in Luebeck and Boston. Current activities include mechanisms of laser tissue effects, optical tissue diagnostics and therapy control, and translational research in optical technologies.

Ralf Brinkmann has a background in quantum optics and lasers and joined the Medical Laser Center Lübeck (MLL) in 1993 after a five years industrial internship. Since 2005 , he is a faculty member at the 
Institute of Biomedical Optics at the University of Lübeck and CEO of MLL since 2011. In addition to the basic research in biophotonics, he is strongly interested in clinical applications, particularly for smart therapeutic laser approaches, and in translational research with industrial partners.

Michael W. Berns is the Arnold and Mabel Beckman professor in the Departments of Biomedical Engineering, Surgery, and Developmental and Cell Biology at the UC Irvine where he is the founding director of the Beckman Laser Institute. He is an adjunct professor of bioengineering and member of the Institute for Engineering in Medicine at the UC San Diego. He has pioneered the use of laser scissors and tweezers to manipulate cells and their organelles.

John A. Parrish is Edward Wigglesworth Distinguished Professor at Harvard Medical School (HMS), and the Chief Executive Officer and
Founder of CIMIT, the Consortia for Improving Medicine with Innovation and Technology, a Boston-based consortium of major teaching hospitals, engineering schools, and research laboratories. He also founded the MGH-Harvard Cutaneous Biology Research Center. A graduate of Duke University and Yale University School of Medicine. He has over 300 publications including seven books.

Gabriela Apiou-Sbirlea is an assistant professor at Harvard Medical School, director of Translational Research Core at Wellman Center for Photomedicine and director of Translation Research Training and Development at Mass General Research Institute. She received her $\mathrm{PhD}$ in biomedical engineering and Habilitation a Diriger des Recherches in health and life sciences in France. She has over 20 years of experience in performing and directing biomedical research in both industry and academia internationally. 